\title{
Caring for who Cares The Family Caregiver Burden
}

Mariana Lázaro', Luísa Gil ${ }^{1}$, Ana Margarida Mota ${ }^{1}$,Filomena Bacelar ${ }^{1}$, Alice Nobre ${ }^{1}$

${ }^{1}$ Centro Hospitalar Psiquiátrico de Lisboa

\section{Objectives}

- Understand the experience of family caregivers of individuals with mental illness;

- Explore what helps family caregivers sustaining their caring role

\section{Backgroud}

Families have been the mainstay of caregiving for people with mental illness. Family caregivers take care of the patient's daily needs, identify the early signs of illness, relapse and deterioration, help the patient in accessing services, supervise treatment and offer emotional support to the patient.

\section{Materials and Methods}

Non-systematic literature review with the keywords "family caregivers", "burden" and "mental illness". Presentation of a case report.

\section{Results and Conclusion}

Case report: we present a case report of an 81 years old female who is the main caregiver of her husband - a patient diagnosed with dementia.

She takes care of her husband and is responsible for all the daily live routines, and is very afraid of being a burden to the family.

After a few years of being the main caregiver, she develops depressive symptomatology, characterized by depressive mood, anxiety, low self-esteem and insomnia.

She starts psychiatry appointments, and gets antidepressive treatment. After feeling better she develops an action plan with her son, in order to get more support for daily activities, for her husband-related responsibilities, and to have free time for leisure activities (like kniting and watching television programs).

Caring for someone with psychiatric illness is associated with a higher level of stress than caring for someone with functional impairment from other chronic medical illnesses WHY?

- Varied nature of psychiatric illness and the inappropriate behavior of the psychiatrically ill;

- The nature of illness may prevent the patient from understanding the caregiver, and in some cases, the patient may even suspect the motives of the caregiver;

- The varied mood swings, disturbed sleep pattern, aggressive and violent outbursts are some other aspects of illness that may further worsen the caregiver's burden.
Potential consequences of caregiver burden:

- Mental-health problems (e.g., depression, anxiety);

- Physical health deterioration (e.g., diabetes);

- Other negative effects (e.g., family dysfunction, social isolation, and financial problems).

Risk Factors for family caregiver's burden:

- Self-reported depression;

- Being over 60 years of age;

- Receiving no help with caregiving;

- Days of contact per week;

- Having other family members needing care.

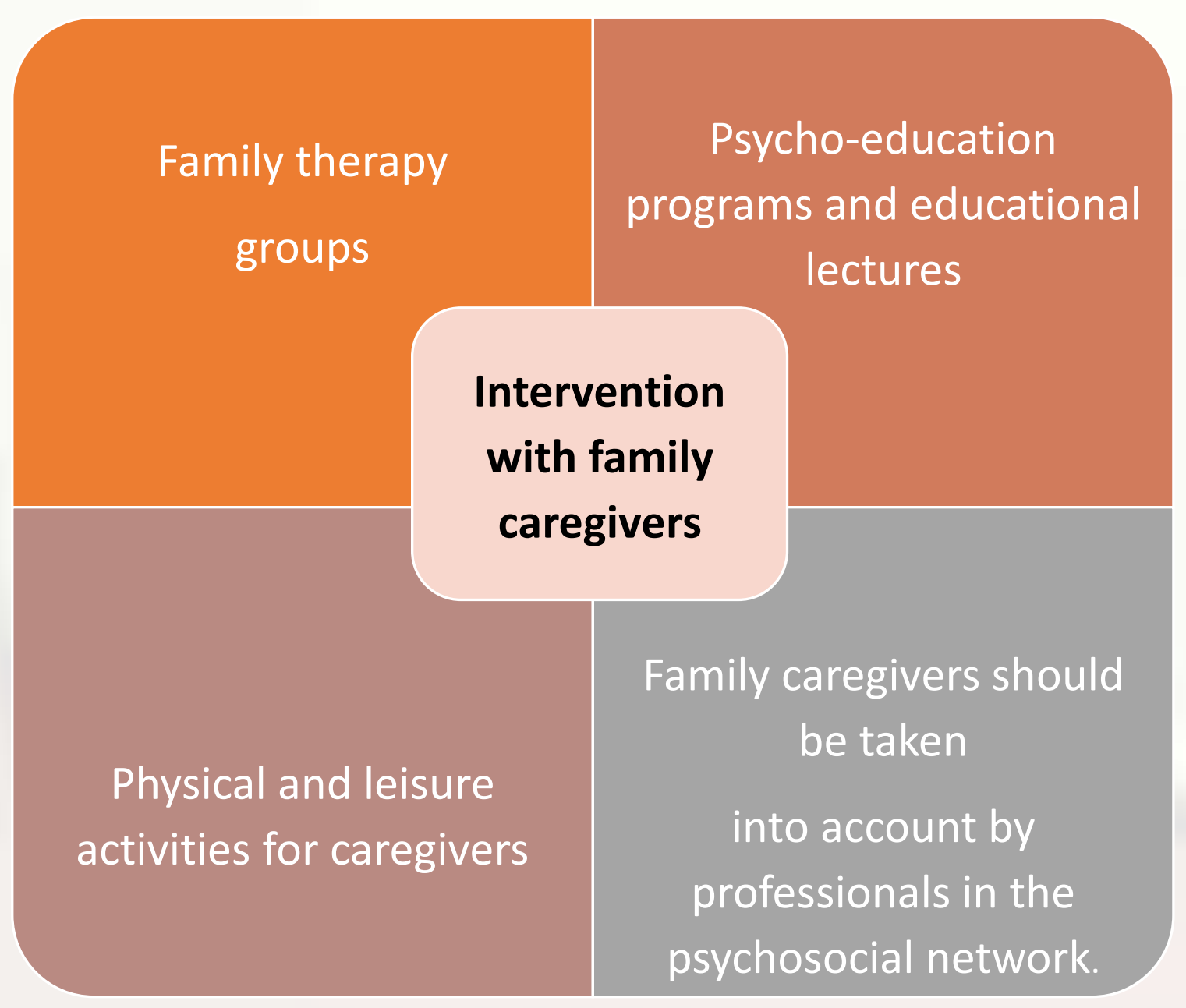

Bibliography: Souza et al, Factors associated with the burden of family caregivers of patients with mental disorders: a cross-sectional study, BMC Psychiatry (2017) 17:353 Ampalam, P. et al, A comparative study of caregiver burden in psychiatric illness and chronic medical illness. Indian J Psychiatry. 2012; 54(3):239-43. 